This article has been updated from its originally published version to correct the title.

Neurosurg Focus 36 (2):E8, 2014

(C)AANS, 2014

\title{
Label-free microscopic assessment of glioblastoma biopsy specimens prior to biobanking
}

\author{
*Joseph Georges, B.S. ${ }^{1,6}$ Aqib Zehri, B.S. ${ }^{1,7}$ Elizabeth Carlson, B.S., ${ }^{5}$ \\ Joshua Nichols, B.A., ${ }^{4}$ Michael A. Mooney, M.D. ${ }^{2}$ Nikolay L. Martirosyan, M.D. ${ }^{1,8}$ \\ Layla Ghaffari, ${ }^{5}$ M. Yashar S. Kalani, M.D., Ph.D., ${ }^{2}$ Jennifer Eschbacher, M.D., ${ }^{3}$ \\ Burt Feuerstein, M.D., Ph.D., ${ }^{7}$ Trent Anderson, Ph.D. ${ }^{4}$ Mark C. Preul, M.D. ${ }^{1}$ \\ Kendall Van Keuren-Jensen, Ph.D., 5 and Peter Nakaji, M.D. ${ }^{2}$
}

${ }^{1}$ Neurosurgery Research Laboratory, ${ }^{2}$ Division of Neurological Surgery, and ${ }^{3}$ Division of Neuropathology, Barrow Neurological Institute, St. Joseph's Hospital and Medical Center, Phoenix; ${ }^{4}$ Department of Basic Medical Sciences, The University of Arizona College of Medicine, Phoenix; ${ }^{5}$ Neurogenomics Division, Translational Genomics Research Institute, Phoenix; ${ }^{6}$ School of Life Sciences, Arizona State University, Tempe; ${ }^{7}$ College of Medicine, The University of Arizona, Phoenix; and ${ }^{8}$ Division of Neurosurgery, Department of Surgery, The University of Arizona, Tucson, Arizona

\begin{abstract}
Glioblastoma is the most common primary brain tumor with a median 12- to 15-month patient survival. Improving patient survival involves better understanding the biological mechanisms of glioblastoma tumorigenesis and seeking targeted molecular therapies. Central to furthering these advances is the collection and storage of surgical biopsies (biobanking) for research. This paper addresses an imaging modality, confocal reflectance microscopy (CRM), for safely screening glioblastoma biopsy samples prior to biobanking to increase the quality of tissue provided for research and clinical trials. These data indicate that CRM can immediately identify cellularity of tissue biopsies from animal models of glioblastoma. When screening fresh human biopsy samples, CRM can differentiate a cellular glioblastoma biopsy from a necrotic biopsy without altering DNA, RNA, or protein expression of sampled tissue. These data illustrate CRM's potential for rapidly and safely screening clinical biopsy samples prior to biobanking, which demonstrates its potential as an effective screening technique that can improve the quality of tissue biobanked for patients with glioblastoma.

(http://thejns.org/doi/abs/10.3171/2013.11.FOCUS13478)
\end{abstract}

KEY WORDS
biopsy glioblastoma $\quad$ confocal reflectance microscopy $\quad$ biobank

$\mathrm{E}$ ACH year, more than 22,000 Americans are diagnosed with high-grade gliomas. More than half of these brain tumors are glioblastomas, the most aggressive and essentially noncurable form of this disease. ${ }^{3}$ Standard treatment for newly diagnosed glioblastoma is resection followed by ionizing radiation and chemotherapy. ${ }^{11}$ However, current therapeutic approaches provide minimal survival benefit, with median survival remaining formidably at 12 months and 2-year survival remaining less than $30 \%{ }^{8,16}$

A key component to improving patient survival involves a better understanding of the biological mechanisms in tumor formation and seeking targeted molecular therapies..$^{13}$ With recent advances in medical genetics,

\footnotetext{
Abbreviations used in this paper: $\mathrm{Akt}=$ protein kinase $\mathrm{B} ; \mathrm{CRM}$ $=$ confocal reflectance microscopy; DAPI $=4,6$-diamidino-2-phenylindole dihydrochloride; GAPDH = glyceraldehyde-3-phosphatedehydrogenase; RIN = RNA integrity number.

* Mr. Georges and Mr. Zehri contributed equally to this work.
}

computational biology, and biotechnology, novel molecular approaches such as immunotherapy, vaccine therapy, and gene therapy are being extensively explored in treating brain tumors. Furthering these advances requires collecting surgical biopsy specimens (biobanking) to study gliomagenesis and to assess a patient's eligibility for potentially life-prolonging clinical trials. Unfortunately, due to the necrotic features of malignant gliomas and our inability to assess tissue prior to biobanking, a large portion of biobanked glioblastoma samples lack appropriate cellularity to be used in these two research arenas. A method for safely screening tissue biopsies prior to biobanking is needed to increase the quality of tissue provided for research and clinical trials.

Confocal reflectance microscopy (CRM) is an optical imaging modality that can rapidly assess tissue without physical manipulation or application of exogenous contrast agents. ${ }^{1,17}$ In CRM, a laser is raster-scanned across a specimen without generating a detectable Stokes shift. Photons from the laser are scattered back toward 


\section{J. Georges et al.}

the objective and passed through a confocal aperture. This allows multiple optical sections to be collected from a sample without physical sectioning. When applied to thick tissue samples, CRM can identify individual cells and structural components within the tissue. ${ }^{1}$ Compared with other optical sectioning techniques such as fluorescence confocal microscopy, structured illumination, or nonlinear microscopy, CRM introduces a fraction of the energy into tissue samples that is encountered with these other techniques. Thus, CRM is theoretically less likely to alter tissue characteristics by generation of free radicals or thermal energy.

We hypothesize that CRM will provide a safe and rapid means for screening glioblastoma tissue prior to biobanking. In this study we used CRM to assess tissue cellularity from rodent glioma models, and subsequently evaluated the molecular integrity of tissue imaged with CRM. Lastly, we assessed CRM on clinical samples with a pathology-based CRM system. Our data illustrate CRM's potential for screening clinical biopsy samples prior to biobanking, which can augment information gained from resected tissue for molecular and translational research and for determining the eligibility of patients with glioblastoma for enrollment into clinical trials. Although numerous applications for intraoperative CRM exist, the goal of this study is to determine its efficacy as a rapid screening technique that will improve the quality of tissues collected and biobanked for glioblastoma research.

\section{Methods}

\section{Intracranial Implantation}

Nude rats were acquired from Charles River Laboratories. Five rats were anesthetized by intramuscular injection of a mixture of $10 \mathrm{mg} / \mathrm{kg}$ xylazine and $80 \mathrm{mg} / \mathrm{kg}$ ketamine (Wyeth) and placed in a small animal stereotactic headframe (Model 900, David Kopf Instruments). A 10 -mm incision was made starting between the animal's eyes to expose bregma. A bur hole was made $3.5 \mathrm{~mm}$ lateral to bregma. Human glioma cells (U251; ATCC) were infused at a depth of $4.5 \mathrm{~mm}$ below the surface of the brain after the syringe (Hamilton) was advanced $5.0 \mathrm{~mm}$ to create a $0.5-\mathrm{mm}$ pocket. The cell suspension was infused using a UMP3-1 UltraMicroPump microinjector (WPI) set to a volume of $10 \mu \mathrm{l}$ with an infusion rate of $3.00 \mu \mathrm{l} / \mathrm{min}$. The needle was withdrawn 2 minutes after the injection to minimize backflow of the cell suspension. The bur hole was covered with bone wax and the skin incision was sutured prior to the rats emerging from anesthesia. All animal procedures were performed according to principles outlined in the NIH Guide for the Care and Use of Laboratory Animals.

\section{Rodent Tissue}

Twenty-eight days after implantation, rodents with xenografts were deeply anesthetized using xylazine and ketamine (as described above), and whole brain specimens were collected. Coronal slices (350 $\mu \mathrm{m}$ thick) were cut from the cerebrum on a Leica VT1200 vibratome and placed in artificial CSF containing the following (in mM): $126 \mathrm{NaCl}, 26 \mathrm{NaHCO}_{3}, 2.5 \mathrm{KCl}, 1.25 \mathrm{NaH}_{2} \mathrm{PO}_{4}$,
$2 \mathrm{MgSO}_{4}, 2 \mathrm{CaCl}_{2}$ and 10 glucose, $\mathrm{pH}$ 7.4). Slices were then fixed in $4 \%$ paraformaldehyde at $4{ }^{\circ} \mathrm{C}$ overnight and washed 3 times with phosphate-buffered saline. Three tumor-containing slices per animal were incubated with DAPI for 45 minutes at room temperature, rinsed 3 times with phosphate-buffered saline, and placed into no. 1.5 glass-bottom dishes (MatTek) for imaging. A coefficient of determination analysis was used to compare cells identified with CRM to cells labeled with DAPI.

The 2 rodents used for molecular experiments were deeply anesthetized using xylazine and ketamine and rapidly decapitated. Whole brains were placed in ice-cold artificial CSF and sectioned into 1-mm coronal sections using a rodent brain block. The cerebrum from each section was blocked into 4 equivalent sections. Two sections were immediately frozen in liquid nitrogen for reference. At 15, 30, 45, 60, 90, and 120 minutes, 2 sections were placed into glass-bottom dishes. At each time point, the cortex, corpus callosum, and caudate/putamen from 1 section was imaged with CRM. As a control, 1 section was simultaneously placed on the stage of the microscope but not imaged. Next, each section was frozen in liquid nitrogen for assessment of DNA, RNA, and protein.

\section{Imaging}

All samples were imaged in uncoated no. 1.5 glassbottom dishes. Confocal reflectance microscopy was conducted with a Zeiss inverted 710 laser-scanning confocal microscope equipped with a $\times 40 / 1.2$ numerical aperture water emersion objective. For reflectance imaging, a 633$\mathrm{nm}$ diode laser was raster scanned across the sample, and reflected photons were collected by tuning the emission filters to allow photons with the same wavelength of the incident laser passage to the photomultiplier tube. For DAPI imaging, samples were excited with a 405-nm diode laser and 430-490 nm emission was collected. The confocal aperture was set to 1 Airy unit for all imaging. The laser and gain values were set to fill the dynamic range of the photomultiplier tube, and the frame size was set to sample at Nyquist. Images were collected in 12-bit format absent of nonlinear processing.

\section{DNA Isolation and Analysis}

DNA was isolated from brain tissue using the QIAamp DNA Mini (Qiagen), per the manufacturer's instructions. DNA was quantified using the Nanodrop Spectrophotometer (Thermo Scientific). Samples were loaded in equal concentrations (100 ng) in a $1 \%$ agarose gel with ethidium bromide and imaged on an Alpha Imager.

\section{RNA Isolation and Analysis}

Tissue was homogenized in $500 \mu \mathrm{l}$ of Ambion's Cell Disruption Buffer (Life Technologies) and subsequently isolated using Ambion's mirVana Paris kit (Life Technologies), per the manufacturer's instructions. RNA concentrations were determined using the Nanodrop Spectrophotometer (Thermo Scientific), which gave dilutions necessary to remain within the dynamic range of the Bioanalyzer. The integrity of the RNA was assessed using Agilent 2100 Bioanalyzer Nanochips (Agilent Technologies). 


\section{Confocal reflectance microscopy assessment of glioblastoma}

\section{Western Blot Analysis}

Frozen tissue was sectioned on dry ice and designated for protein, RNA, or DNA analysis. Protein lysate was made by placing brain sections into $750 \mu \mathrm{l}$ of Ambion's Cell Disruption Buffer (Life Technologies), triturated using RNase-free pipettes, and sonicated using Covaris Sonolab at 2\%-5\% for 5 seconds, $2 \%-20 \%$ for $15 \mathrm{sec}-$ onds, $2 \%-20 \%$ for 15 seconds, and $2 \%-5 \%$ for 5 seconds (Covaris Inc).

Protein concentrations were quantified by bicinchoninic acid assay, and $18 \mu \mathrm{g} /$ lane was loaded in 4\%-12\% Bis-Tris gels and run using NuPage electrophoresis reagents (Invitrogen). Protein was transferred onto Novex nitrocellulose membrane (Invitrogen) and thereafter incubated for 1 hour in a blocking solution consisting of 5\% bovine serum albumin (Sigma Aldrich) in tris-buffered saline with $0.1 \%$ Tween (Thermo Fisher Scientific). Primary antibodies were incubated for 12 hours at $4^{\circ} \mathrm{C}$ while secondary horseradish peroxidase-conjugated antibodies were incubated for 1 hour at room temperature. Blots were probed for protein kinase B (Akt; 1:1000, Cell Signaling) and GAPDH (1:60,000, Millipore). Horseradish peroxidase-conjugated secondary antibodies were anti-rabbit (1:2000, Cell Signaling) and anti-chicken (Millipore).

Blots were developed by using Pierce SuperSignal Chemiluminescent Substrate (Thermo Fisher Scientific) per the manufacturer's instructions. Protein signal was detected on radiographic film (General Electric).

\section{Statistical Analysis}

A coefficient of correlation $\left(\mathrm{R}^{2}\right.$ value) was determined between DAPI-stained nuclei and nuclei detected by CRM using Graphpad Prism. Differences were considered statistically significant for probability $<0.05$. The Agilent 2100 Bioanalyzer provided an RNA integrity number (RIN), calculated algorithmically by including the 28s/18s (ribosomal subunit) ribosomal RNA bands, the region before the peaks, signal areas, and intensities. An elevated threshold baseline and a decreased 28s/18s ratio are both indicative of RNA degradation, while high 28 s and 18s ribosomal RNA peaks as well as a small amount of 5s RNA or an RNA number greater than 7.5 are indicative of intact RNA.7 Image $\mathrm{J}$ was used to determine density (intensity) of bands on a Western blot. Data analysis consisted of determining relative density. Results were expressed as means and mean square error (SEM) data with normal distribution that were compared by 1-way ANOVA and a Student t-test.

\section{Clinical Samples}

The clinical research was approved by the Institutional Review Board of St. Joseph's Hospital and Medical Center and Barrow Neurological Institute, where all surgery was performed. Preoperatively, patients signed an informed consent form for participation. Samples (mean size $4 \mathrm{~mm} \times 2 \mathrm{~mm} \times 2 \mathrm{~mm}$ ) were obtained at the time of craniotomy from within the tumor mass at a location determined to be safe by the surgeon. Tissue samples were placed in ice-cold artificial CSF and transported from the operating room to the pathology-based CRM system.
There, the samples were immediately placed in no. 1.5 glass-bottom dishes and imaged. Investigators conducting imaging experiments were unaware of the pathological diagnosis at the time of imaging. Final diagnosis was determined by traditional immunohistochemical analysis and paraffin-embedded $\mathrm{H} \& \mathrm{E}$ staining of the sampled tissue. For the purpose of comparisons, the histopathological diagnosis made by a board-certified neuropathologist (J.E.) was accepted as the final diagnosis.

\section{Results}

\section{Differentiating Neoplastic Cellular Tumor From Acellular} Tissue

To investigate the potential of reflectance imaging to identify tissue cellularity, we first imaged normal rat brain. We found that CRM adequately contrasted normal brain cytoarchitecture, such as cell bodies and axons, as well as blood vessels (Fig. 1). This prompted us to test the utility of CRM to differentiate cellular and acellular tumor biopsy regions by imaging acute slices generated from rodent glioblastoma xenografts. We incubated slices with DAPI to label all cell nuclei and we subsequently imaged the slices with CRM and laser-scanning confocal microscopy ( $\mathrm{n}=15$ slices from 5 animals). We collected 5 images per acute slice and compared cells identified with CRM to cells identified with DAPI. We found CRM provided definitive contrast between cell nuclei, cytoplasm, and extracellular tissue. Within tumor regions, CRM provided contrast to visualize hypercellular tumor regions (Fig. 2A-C) and relatively acellular peritumoral regions with isolated cell populations (Fig. 2E-G). In hypercellular $\left(r^{2}=0.97\right)$ and acellular regions $\left(r^{2}=0.098\right)$ we found CRM contrast strongly correlated with cells labeled with DAPI (Fig. 2D and H).

\section{Effect of CRM on the Molecular Characteristics of Examined Tissue}

To determine if CRM alters the molecular characteristics of tissue, we compared DNA, RNA, and protein from tissue imaged with CRM to tissue immediately frozen for analysis and tissue that had reflectance imaging and was left out for varying amounts of time. Although the typical time from resection to reception in pathology and assessment using CRM typically takes 15 minutes, we tested for as long as 180 minutes postresection. Neoplastic tissues are heterogeneous in terms of cellularity and gene and protein expression and may yield interspecimen molecular variability. Therefore, we conducted these experiments on control tissue harvested from rodent normal brain.

DNA quality was assessed in CRM-imaged samples, which showed no differences compared with immediately frozen controls. Discrete DNA bands were detectable up to 180 minutes after extraction (Fig. 3B), suggesting no degradation of DNA elements. An RIN was generated to determine the integrity of isolated RNA. An RIN of 1 suggests strong degradation while an RIN greater than 8 suggest minimal degradation. ${ }^{3}$ The RIN was comparably the same between control and CRM-imaged groups (Fig. 

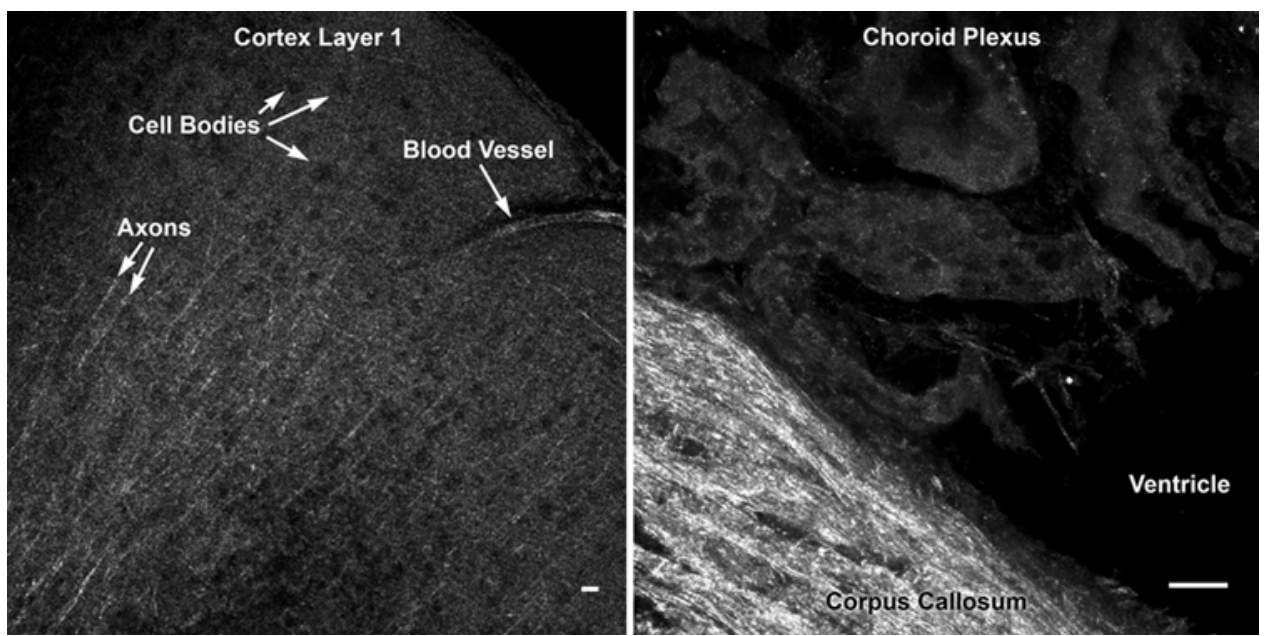

Fig. 1. Acute slices of normal rat brain from the cortex (left) and ventricle and corpus callosum (right) shown using contrastfree reflectance imaging. Left: Confocal reflectance microscopy contrasts cell bodies, axons, and blood vessels in normal rat brain. Cell bodies appear as multiple hypointense circular regions within the tissue. Note the typical lack of cell bodies contrasted in Layer 1 of the cortex. Myelinated axons are visualized as hyperintense fibers extending from cell bodies. Right: White matter tracts in the corpus callosum generate a hyperintense reflectance signal. Individual cell bodies are contrasted in the choroid plexus. A lack of signal is appreciated from the fluid-filled ventricle. Bar $=20 \mu \mathrm{m}$.

3C), indicating no effect of CRM on RNA integrity of imaged samples. RNA integrity remained relatively the same up to 120 minutes after biopsy. There was a slight decrease after 120 minutes after biopsy in RIN value that was similar for both the control and CRM tissue, likely due to RNases within the tissue over time. Protein kinase $\mathrm{B}$, a protein involved in glioblastoma pathogenesis, was examined for potential damage after imaging with CRM.
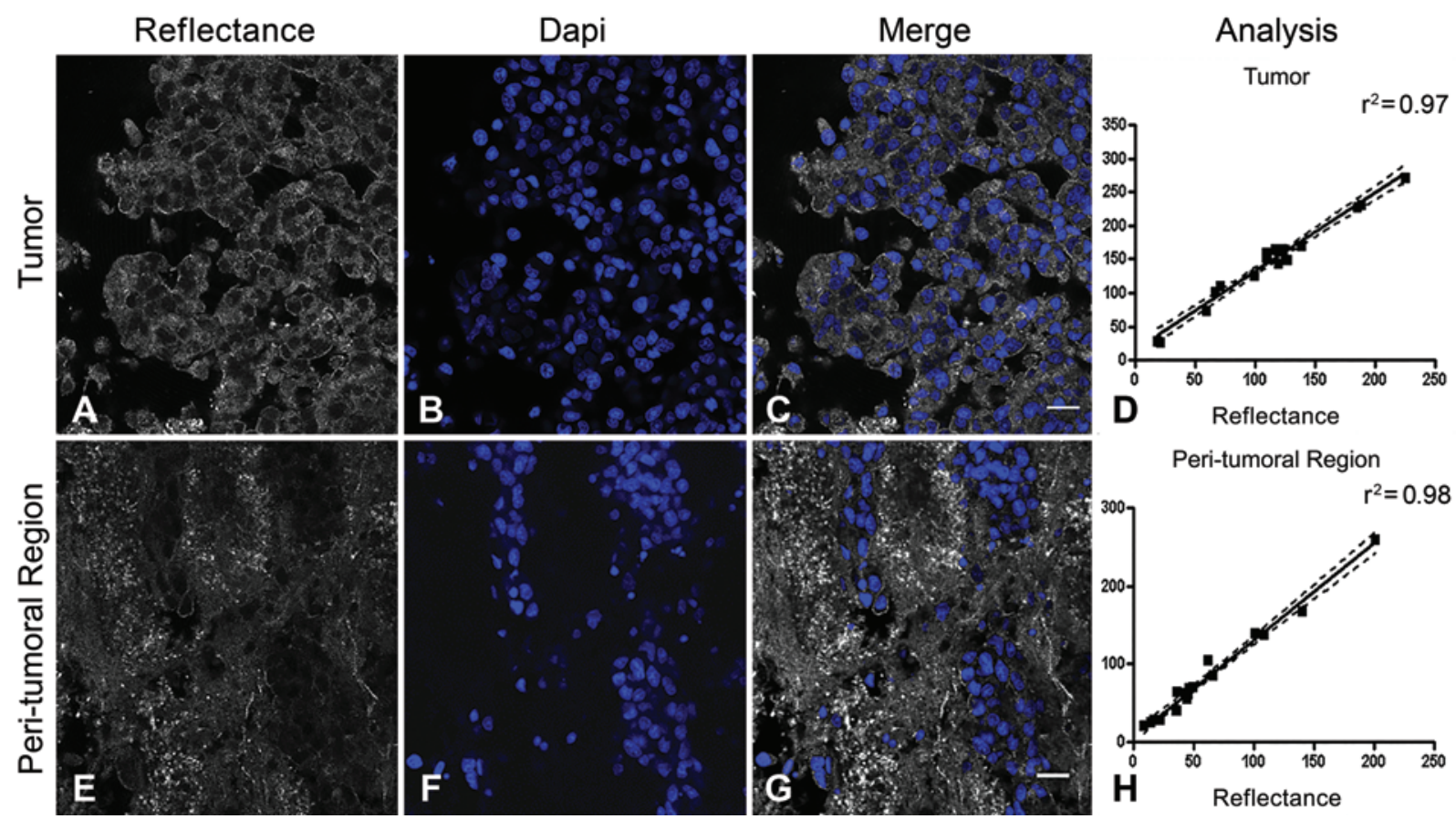

Fig. 2. Confocal reflectance imaging identifies cellular tumor in rodent acute slices. A: Reflectance image of rodent xenograft tumor region; note hypointense cell nuclei. B: DAPI stain of identical tumor region identifies all cells in the field of view. C: Merged image shows location of cells contrasted by reflectance in comparison with cells labeled with DAPI. Bar $=20$ $u m$. D: Plot of coefficient of determination and $95 \%$ Cls for tumor cells identified by reflectance imaging $\left(r^{2}=0.97\right)$. The solid line is the best fit slope. The dotted lines mark the $95 \%$ Cls. The dots are the counts from each region of interest. The y axis is actual number of cells (per DAPI staining), and the $x$ axis shows cells counted by reflectance imaging. E: Reflectance image of cellular tumor and adjacent acellular region from rodent xenograft; note isolated cell populations. F: Fluorescence confocal image of identical region labeled with DAPI. G: Merged image of reflectance and fluorescence images from tumor and peritumoral tissue interface. $\mathrm{H}$ : Plot of coefficient of determination and $95 \% \mathrm{Cls}$ for cells identified by reflectance imaging at tumor and acellular tissue interface $\left(r^{2}=0.98\right)$. Bar $=20 \mu \mathrm{m}$. 

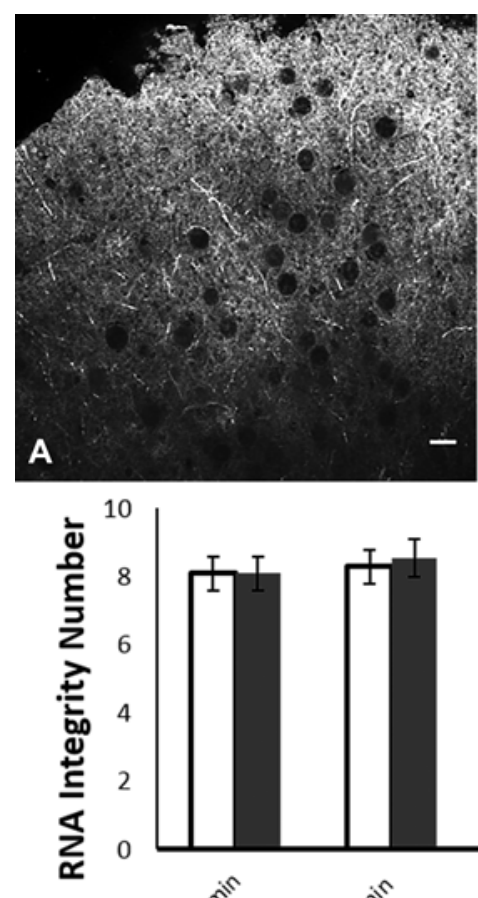

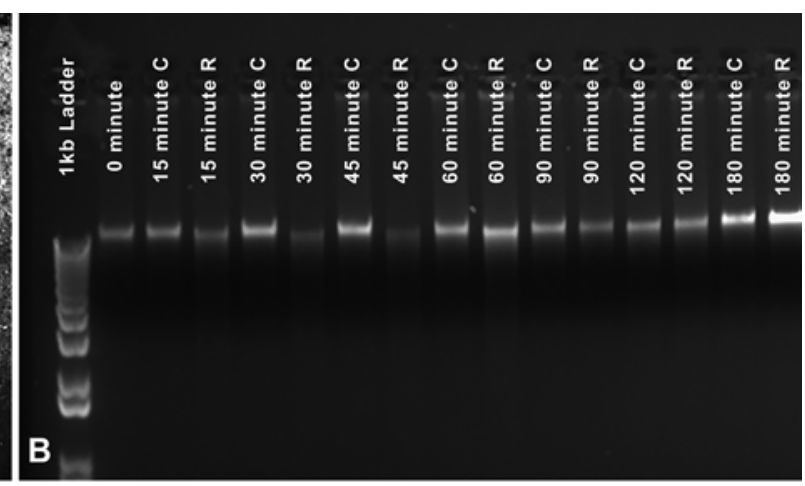

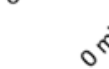

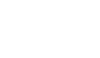

Control
Reflectance

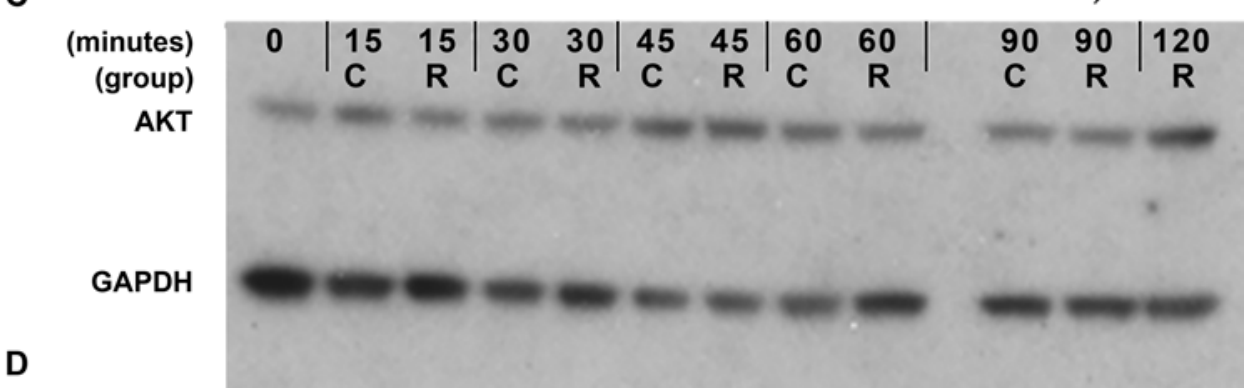

FIG. 3. Images showing CRM does not alter DNA, RNA, or protein of examined tissue in rodent brain. A: Confocal reflectance microscopy image of fresh brain tissue at the cerebral cortex obtained 90 minutes after excision. Bar $=20 \mu \mathrm{m}$. B: Gel electrophoresis of genomic DNA extracted from control $(C)$ and CRM-imaged biopsies (R); note similar bands across the time course between imaged and control biopsies. C: Time course comparison of RNA integrity for all imaged and control samples. Note similar RNA integrity for all samples. D: Western blot analysis showing expression of Akt over a 120-minute time course. The protein signal did not degrade over the time course or when exposed to CRM. The GAPDH loading control shows a similar amount of protein lysate was loaded into each well of the Western blot.

Western blot analysis of extracted tissue showed that up to 120 minutes after extraction, discrete Akt bands were detectable and contained similar density to control samples (Fig. 3D).

\section{Differentiating Human Cellular From Acellular Brain Tumor Biopsies}

To test the ability of CRM to differentiate cellular tumor from acellular tissue samples in a clinical setting, we imaged 2 fresh human brain tumor biopsy specimens: 1 yielding radiation necrosis tissue and 1 with known glioblastoma cellular tissue. Tissue samples were placed in ice-cold artificial CSF and imaged with CRM. Imaging time per sample was less than 2 minutes. Samples were then compared with final histopathological diagnosis.

Similar to our findings in rodent xenografts, we found that CRM contrasted cellular regions from acellular regions in human biopsy samples. Tissue samples identified as cellular with CRM were found to be cellular with subsequent histopathological assessment (Fig. 4A and C). Confocal reflectance microscopy correctly identified acellular regions in necrotic tissue samples (Fig. 4B and D).

\section{Discussion}

Our data illustrate the utility of CRM as a safe and rapid technique for identifying the cellularity of glioma tissue prior to biobanking. This imaging modality can be immediately used on fresh tissue samples without application of exogenous contrast agents and without altering the molecular characteristics of examined tissue. Confocal reflectance microscopy can provide a much-needed tool for neurosurgery and neuropathology teams by maximizing the quality of tissue samples collected during resection. Although other imaging modalities may provide excellent images, their greater energy and the possible 

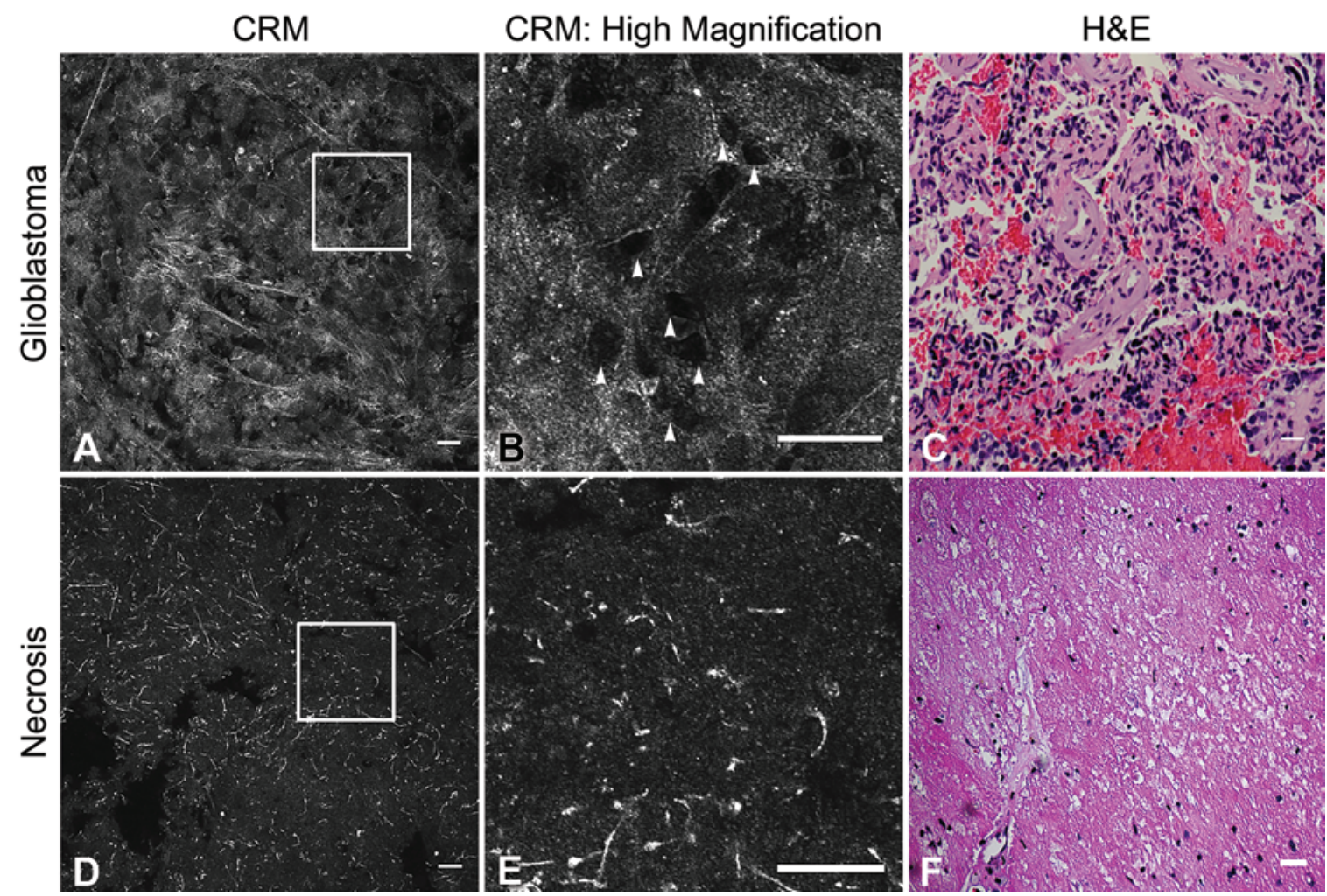

FIG. 4. Reflectance imaging immediately identifies cellular and necrotic fresh human brain tumor biopsies. A: Low-magnification CRM image of ex vivo human glioblastoma biopsy specimen imaged with CRM. B: High magnification of cellular region shown in panel A. Note arrowheads identifying cells within the region. C: Corresponding H \& E image from panel B. D: Low magnification image of ex vivo necrotic biopsy specimen imaged with CRM. E: High magnification of inset in panel D. Note the lack of distinct cells. F: Corresponding $\mathrm{H} \& \mathrm{E}$ image from panel $\mathrm{E}$. All bars $=20 \mu \mathrm{m}$.

need to use exogenous fluorophores could affect future molecular analysis of the tissues. ${ }^{12}$

We found CRM did not alter the DNA, RNA, or protein that could be extracted and quantified from tissue biopsy specimens screened up to 2 hours after resection. Confocal reflectance microscopy images collected from these samples could be digitally stored and potentially recalled with biobanked specimens. This metadata could prove advantageous to researchers who want more information about tumor morphology or cellularity before they choose it for laborious or costly analyses.

Confocal reflectance microscopy provides cellular and subcellular information. We ascertain CRM to have diagnostic utility, as many images in this study revealed distinct morphological details of glioblastoma such as cellularity, vasculature, and necrosis (Figs. 1, 2, 3A, and 4) typically identified with traditional histopathological $\mathrm{H} \&$ E staining.

Many translational neurooncology studies rely on human glioblastoma tissue samples that appropriately represent an original tumor. Unintentional utilization of necrotic or nonrepresentative tissue samples can lead to erroneous and biased results that confound molecular and genetic experiments. In studies that advance to clinical trials, patient biopsies are often screened to determine eligibility for a targeted clinical trial. Utilization of CRM can ensure that patients are not mistakenly excluded from these trials after standard therapy has failed. Therefore, screening for high-quality tissue specimens with CRM can facilitate the advancement of our knowledge of glioma biology and can ensure qualified patients receive potential life-prolonging therapies and can enroll in appropriate clinical trials. ${ }^{14}$

Current limitations of CRM include limited imaging depth penetration. With our imaging parameters, CRM could assess tissue from the surface to a depth of 200-300 $\mu \mathrm{m}$. However, we did not find that this depth limitation altered our assessment of tissues in our study. At present, few pathology departments contain the imaging hardware or personnel required to screen tissue biopsies with CRM. We have a full-scale laboratory Zeiss LSM710 microscope located for research purposes in the hospital's clinical pathology department where operating room specimens are routinely received, but equivalent equipment is rarely found in the pathology departments of most hospitals. However, modernization of pathology departments may include addition of confocal microscopes and other systems capable of CRM that will allow the screening of samples within 2 hours. Lastly, ex vivo CRM is limited by the ability to only assess tissue that is intraoperatively selected to represent tumor. A handheld intraoperative CRM device could potentially overcome sampling error and allow assessment of tissue samples prior to resection.

Past studies have shown the utility of CRM technology in providing cellular and subcellular detail, specifi- 


\section{Confocal reflectance microscopy assessment of glioblastoma}

cally in diagnosing dermatological conditions, identifying neoplastic tissue and margins, and assessing diseased and normal liver tissue. , $2,4-6,9,10,15,18$ Our study is the first to assess human brain tumor tissue purely with CRM. By quantifying DNA, RNA, and protein, we also demonstrate for the first time the ability of CRM to preserve the molecular integrity of tissue biopsies.

\section{Conclusions}

Confocal reflectance microscopy can screen brain tumor tissue cellularity for inclusion into biobanks while preserving molecular integrity of tissue samples. Confocal reflectance microscopy provides a rapid imaging modality that can accurately provide ex vivo morphological information in animal models and fresh human biopsies. In comparison with traditional histopathological methods, this technique does not rely on exogenous dyes or fixation and sectioning. Furthermore, this technique preserves the DNA, RNA, and protein characteristics of tissues, allowing further analysis of imaged specimens. Future technical developments of CRM include utilization of a handheld confocal endomicroscope for imaging, which would allow rapid and safe histopathological assessments in vivo. Further applications of CRM may include rapid ex vivo and in vivo examination of brain tumors in addition to glioblastoma. This technique ensures that high quality specimens are biobanked for future molecular studies of tumor samples and for assessing patient eligibility for clinical trials based on tumor characteristics.

\section{Disclosure}

The authors report no conflict of interest concerning the materials or methods used in this study or the findings specified in this paper.

Author contributions to the study and manuscript preparation include the following. Conception and design: Nakaji, Georges, Zehri, Eschbacher, Feuerstein, Preul, Keuren-Jensen. Acquisition of data: Georges, Zehri, Carlson, Nichols, Martirosyan, Ghaffari, Eschbacher, Anderson, Keuren-Jensen. Analysis and interpretation of data: Georges, Zehri, Carlson, Ghaffari, Eschbacher, KeurenJensen. Drafting the article: Georges, Zehri. Critically revising the article: Nakaji, Mooney, Kalani, Eschbacher, Feuerstein, Preul, Keuren-Jensen. Reviewed submitted version of manuscript: Nakaji, Zehri, Preul, Keuren-Jensen. Statistical analysis: Georges, Carlson, Keuren-Jensen. Administrative/technical/material support: Nakaji, Feuerstein, Anderson, Preul, Keuren-Jensen. Study supervision: Nakaji, Eschbacher, Feuerstein, Anderson, Preul, Keuren-Jensen.

\section{References}

1. Campo-Ruiz V, Lauwers GY, Anderson RR, Delgado-Baeza E, González S: In vivo and ex vivo virtual biopsy of the liver with near-infrared, reflectance confocal microscopy. Mod Pathol 18:290-300, 2005

2. Campo-Ruiz V, Ochoa ER, Lauwers GY, González S: Evaluation of hepatic histology by near-infrared confocal microscopy: a pilot study. Hum Pathol 33:975-982, 2002

3. Central Brain Tumor Registry of the United States: CBTRUS Statistical Report: Primary Brain and Central Nervous System Tumors Diagnosed in the United States 2004-2006.
(http://www.cbtrus.org/2010-NPCR-SEER/CBTRUS-WEB REPORT-Final-3-2-10.pdf) [Accessed December 16, 2013]

4. Clark AL, Gillenwater AM, Collier TG, Alizadeh-Naderi R, El-Naggar AK, Richards-Kortum RR: Confocal microscopy for real-time detection of oral cavity neoplasia. Clin Cancer Res 9:4714-4721, 2003

5. Curiel-Lewandrowski C, Williams CM, Swindells KJ, Tahan SR, Astner S, Frankenthaler RA, et al: Use of in vivo confocal microscopy in malignant melanoma: an aid in diagnosis and assessment of surgical and nonsurgical therapeutic approaches. Arch Dermatol 140:1127-1132, 2004

6. Drezek RA, Richards-Kortum R, Brewer MA, Feld MS, Pitris C, Ferenczy A, et al: Optical imaging of the cervix. Cancer 98 (9 Suppl):2015-2027, 2003

7. Fleige S, Pfaffl MW: RNA integrity and the effect on the realtime qRT-PCR performance. Mol Aspects Med 27:126-139, 2006

8. Furnari FB, Fenton T, Bachoo RM, Mukasa A, Stommel JM, Stegh A, et al: Malignant astrocytic glioma: genetics, biology, and paths to treatment. Genes Dev 21:2683-2710, 2007

9. González S, Swindells K, Rajadhyaksha M, Torres A: Changing paradigms in dermatology: confocal microscopy in clinical and surgical dermatology. Clin Dermatol 21:359-369, 2003

10. Hicks SP, Swindells KJ, Middelkamp-Hup MA, Sifakis MA, González E, González S: Confocal histopathology of irritant contact dermatitis in vivo and the impact of skin color (black vs white). J Am Acad Dermatol 48:727-734, 2003

11. Lefranc F, Brotchi J, Kiss R: Possible future issues in the treatment of glioblastomas: special emphasis on cell migration and the resistance of migrating glioblastoma cells to apoptosis. J Clin Oncol 23:2411-2422, 2005

12. Liao LD, Tsytsarev V, Delgado-Martínez I, Li ML, Erzurumlu R, Vipin A, et al: Neurovascular coupling: in vivo optical techniques for functional brain imaging. Biomed Eng Online 12:38, 2013

13. Mohyeldin A, Chiocca EA: Gene and viral therapy for glioblastoma: a review of clinical trials and future directions. Cancer J 18:82-88, 2012

14. Rainov NG, Heidecke V: Clinical development of experimental therapies for malignant glioma. Sultan Qaboos Univ Med J 11:5-28, 2011

15. Rajadhyaksha M, González S, Zavislan JM, Anderson RR, Webb RH: In vivo confocal scanning laser microscopy of human skin II: advances in instrumentation and comparison with histology. J Invest Dermatol 113:293-303, 1999

16. Stupp R, Mason WP, van den Bent MJ, Weller M, Fisher B, Taphoorn MJ, et al: Radiotherapy plus concomitant and adjuvant temozolomide for glioblastoma. N Engl J Med 352:987-996, 2005

17. Tilli MT, Cabrera MC, Parrish AR, Torre KM, Sidawy MK, Gallagher AL, et al: Real-time imaging and characterization of human breast tissue by reflectance confocal microscopy. J Biomed Opt 12:051901, 2007

18. White WM, Tearney GJ, Pilch BZ, Fabian RL, Anderson RR, Gaz RD: A novel, noninvasive imaging technique for intraoperative assessment of parathyroid glands: confocal reflectance microscopy. Surgery 128:1088-1101, 2000

Manuscript submitted October 14, 2013.

Accepted November 22, 2013.

Please include this information when citing this paper: DOI: 10.3171/2013.11.FOCUS13478.

Address correspondence to: Peter Nakaji, M.D., c/o Neuroscience Publications, Barrow Neurological Institute, St. Joseph's Hospital and Medical Center, 350 W. Thomas Rd., Phoenix, AZ 85013. email: neuropub@dignityhealth.org. 